\begin{tabular}{|c|l|}
\hline Title & Antiparallel spin Hall current in a bilayer with skew scattering \\
\hline Author(s) & Ishikawa, Toshiya; A kera, Hiroshi \\
\hline Citation & $\begin{array}{l}\text { Physical Review B, 100(12), 125307 } \\
\text { https://doi.org/10.1103/PhysRevB.100.125307 }\end{array}$ \\
\hline Issue Date & 2019-09-20 \\
\hline Doc URL & http://hdl.handle.net/2115/76052 \\
\hline Rights & Copyright (2019) by The A merican Physical Society. \\
\hline Type & article \\
\hline File Information & PhysRevB.100.125307.pdf \\
\hline
\end{tabular}

Instructions for use 


\title{
Antiparallel spin Hall current in a bilayer with skew scattering
}

\author{
Toshiya Ishikawa and Hiroshi Akera $\odot$ \\ Division of Applied Physics, Graduate School and Faculty of Engineering, Hokkaido University, Sapporo, Hokkaido 060-8628, Japan
}

(Received 20 December 2018; revised manuscript received 7 August 2019; published 20 September 2019)

\begin{abstract}
The spin Hall effect due to skew scattering is studied by solving the Boltzmann equation in a bilayer electron system with attractive impurity potentials in one layer and repulsive ones in the other. In such an impurity configuration, directions of the spin Hall current in two decoupled layers are antiparallel. An analytical formula for the magnitude of the antiparallel spin Hall current is derived in the crossover from the decoupled bilayer to the strongly coupled one with no spin Hall current with increasing $\Delta_{\text {SAS }}$, the energy separation between the symmetric and antisymmetric states of the motion perpendicular to the layer. When the impurity potential is short ranged and $\Delta_{\mathrm{SAS}} \ll \varepsilon_{\mathrm{F}}$, with $\varepsilon_{\mathrm{F}}$ being the Fermi energy, the normalized antiparallel spin Hall conductivity is found to be $\left[1+\left(\omega \tau_{p}\right)^{2}\right]^{-1}$, with $\omega=\Delta_{\mathrm{SAS}} / \hbar$ and $\tau_{p}$ being the momentum relaxation time at $\varepsilon_{\mathrm{F}}$. This formula is explained by extending the dynamics for the Hanle effect, which was originally developed for spin, to the pseudospin (layer) degree of freedom. The present finding suggests that the Hanle effect will be useful in understanding the pseudospin dynamics as well as the spin dynamics.
\end{abstract}

DOI: 10.1103/PhysRevB.100.125307

\section{INTRODUCTION}

The spin Hall effect [1-3], the generation of the spin current perpendicular to the electric field by the action of the relativistic spin-orbit interaction (SOI), is one of the major research fields in condensed-matter physics and is also technologically important in spintronics [4] because it provides an efficient method for creating the spin current and the spin polarization. The mechanism of the spin Hall effect is divided into the intrinsic origin (band structure effect) $[5,6]$ and the extrinsic one (impurity effect) [7-13]. The extrinsic spin Hall effect has the advantage in studying the dependence on the dimensionality and the structure of the system since it has been experimentally observed, including its inverse effect, in a wide variety of systems $[14,15]$ and materials [16-18]. In fact, the observation of the extrinsic spin Hall effect, prior to that of the intrinsic spin Hall effect [19], was first made in three-dimensional electron systems [14] and subsequently in two-dimensional electron systems formed in semiconductor quantum-well structures [15]. Further development of the research on the extrinsic spin Hall effect is expected in various quantum-well structures. In this paper we theoretically study the extrinsic spin Hall effect in a bilayer electron system formed in double quantum wells.

Bilayer electron systems in double quantum wells have been shown in the quantum Hall regime to exhibit rich phenomena caused by the additional orbital degree of freedom called the pseudospin [20-27]. In the pseudospin space, the bonding (symmetric) and antibonding (antisymmetric) states are formed and have energy separation $\Delta_{\mathrm{SAS}}$ in the case of a symmetric bilayer (Fig. 1). It was shown experimentally [28-31] and theoretically [32,33] that the bilayer system makes a phase transition between the presence and absence of the quantum Hall effect with changing $\Delta_{\text {SAS }}$ when the total Landau level filling factor $v$ is odd. This phase transition is caused by one role of $\Delta_{\mathrm{SAS}}: \Delta_{\mathrm{SAS}}$ delocalizes the electron wave function between two layers, competing with the electron-electron interaction, which localizes it within one layer.

Now we consider a crossover between the presence and absence of the extrinsic spin Hall effect [34] with changing $\Delta_{\text {SAS }}$ in a bilayer electron system (a typical bilayer electron system is a GaAs/AlGaAs heterostructure with two GaAs quantum wells). If the bilayer has a tailored impurity configuration in which attractive impurity potentials are in one layer and repulsive ones are in the other (in a GaAs well layer, $\mathrm{Si}$ and $\mathrm{Be}$ impurities have attractive and repulsive potentials, respectively [35]), the crossover actually occurs between the decoupled bilayer (negligible $\Delta_{\text {SAS }}$ ) with antiparallel spin Hall current and the strongly coupled bilayer (large $\Delta_{\text {SAS }}$ ) with no spin Hall current since it is known that the direction of the spin Hall current due to the skew scattering [36-38] is opposite between attractive and repulsive impurity potentials [12,39]. However, there remains an important unsolved problem: how the magnitude of the antiparallel spin Hall current changes from full magnitude to zero with increasing $\Delta_{\mathrm{SAS}}$, in particular what determines the value of $\Delta_{\mathrm{SAS}}$ at the crossover. The present system has several energy scales, which can be of the same order of magnitude as $\Delta_{\mathrm{SAS}}$, such as $k_{\mathrm{B}} T$ and the level broadening due to impurity scattering and, in addition, has several timescale candidates to compete with $\hbar / \Delta_{\text {SAS }}$ such as the momentum relaxation time $\tau_{p}$, the inverse of skew-scattering rate, and the pseudospin-dephasing time. Suppose, for example, that the crossover occurs around $\Delta_{\mathrm{SAS}}=k_{\mathrm{B}} T$. This would mean that the crossover can be driven by the change in the electron population in bonding and antibonding states. In such a manner, we uncover one mechanism of the crossover by identifying a quantity which determines the $\Delta_{\text {SAS }}$ value at the crossover.

In the present paper we theoretically investigate the crossover between the decoupled bilayer with antiparallel extrinsic spin Hall current and the strongly coupled bilayer 


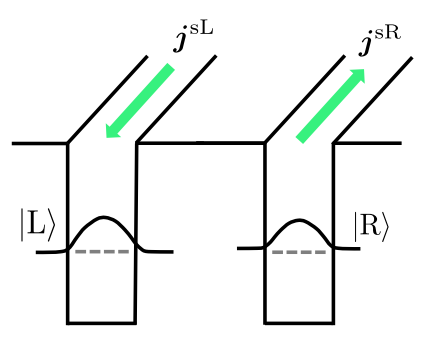

decoupled

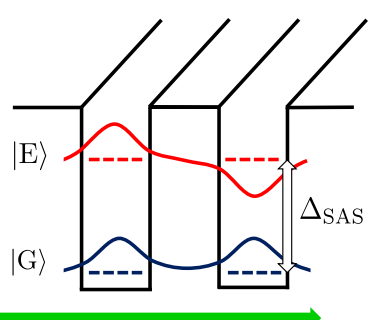

coupled $\Delta_{\mathrm{SAS}}$
FIG. 1. Coupled and decoupled double-quantum-well (DQW) structures. Wave functions for the motion perpendicular to the coupled symmetric DQW are the bonding (symmetric) $|G\rangle$ and antibonding (antisymmetric) $|E\rangle$ states, each of which is composed of wave functions in the left and right wells, $|L\rangle$ and $|R\rangle$. The energy separation $\Delta_{\mathrm{SAS}}$ between $|G\rangle$ and $|E\rangle$ represents the strength of the coupling between $|L\rangle$ and $|R\rangle$. The DQW considered in this paper is assumed to have antiparallel extrinsic spin Hall current, $\boldsymbol{j}^{\mathrm{sL}}=-\boldsymbol{j}^{\mathrm{sR}}$, when it is decoupled.

with vanishing spin Hall current as a function of $\Delta_{\text {SAS }}$ in order to clarify quantities determining the value of $\Delta_{\text {SAS }}$ at the crossover. In addition to the momentum relaxation time and the skew-scattering rate included in the theory of the extrinsic spin Hall effect, we take into account the pseudospindephasing time and the nonzero temperature. Our calculation does not account for the level broadening since the spin current is calculated up to third order with respect to the impurity potential.

We introduce the current carrying both the spin and the pseudospin, which is defined in Eq. (59) by the statistical average of the operator product of the spin, the pseudospin, and the velocity. The antiparallel component of the spin Hall current corresponds to the current carrying the spin perpendicular to the bilayer and the third component of the pseudospin. We calculate such a spin-pseudospin current by analytically solving the Boltzmann equation. In the collision term of the equation we apply the assumption made in the Dyakonov-Perel spin-relaxation theory [40-42] to the case of pseudospin; that is, we neglect the interlayer tunneling described by $\Delta_{\text {SAS }}$ in the collision term and interlayer matrix elements of the impurity potential.

We find, in the case when the Fermi energy is large compared to $k_{\mathrm{B}} T$ and $\Delta_{\mathrm{SAS}}$, that the momentum relaxation time $\tau_{p}$ and the pseudospin-dephasing time are relevant in determining the crossover value of $\Delta_{\mathrm{SAS}}$, while the skew-scattering rate and $k_{\mathrm{B}} T$ are irrelevant. The derived antiparallel spin Hall conductivity, normalized by the value at $\Delta_{\mathrm{SAS}}=0$, is $[1+$ $\left.\left(\omega \tau_{p}\right)^{2}\right]^{-1}$, with $\omega=\Delta_{\text {SAS }} / \hbar$, when the pseudospin dephasing is absent and the impurity potential is short ranged. This formula indicates that the crossover is driven by the competition between the two timescales, the pseudospin-precession period $h / \Delta_{\text {SAS }}$ and the momentum relaxation time $\tau_{p}$. The reduction by the pseudospin precession $\omega$ of the obtained spin Hall conductivity suggests that the present crossover can be regarded as the Hanle effect [43-45] in the pseudospin degree of freedom. In fact, the equation of motion for three pseudospin components of the spin-pseudospin current has the same form as that for three spin components in the Hanle effect [45]. Because the quantity we consider is the current carrying the pseudospin (and the spin), it relaxes via the momentum relaxation as well as the pseudospin dephasing.

The organization of this paper is as follows. Section II describes our Hamiltonian and introduces pseudospin operators and basis vectors, while Sec. III explains the assumptions and approximations which are employed in the following calculation. Section IV derives the Boltzmann equation for the distribution function, the operator in the pseudospin space, in our bilayer system, which includes the skew-scattering component of the collision term and the pseudospin-precession term. Then we rewrite the derived Boltzmann equation using pseudospin components of the distribution operator, each of which is the statistical average of the corresponding pseudospin operator. The solution for the distribution operator in the linear response to the applied in-plane electric field, which is obtained in Sec. V, is employed to obtain the analytical formula for pseudospin components of the spin Hall conductivity in Sec. VI. In this section we show that the third pseudospin component of the spin Hall conductivity exhibits a decrease with increasing pseudospin-precession frequency and that this reduction is explained by the pseudospin-current analog of the Hanle effect. We also propose a possible experiment to confirm the reduction of the third component due to the pseudospin precession by introducing the potential offset between two layers. In Sec. VII, conclusions are given.

\section{HAMILTONIAN}

Our system is a double-quantum-well structure with translational symmetry in the $x y$ plane. We consider conductionband electron states within the effective-mass approximation (see [46] for the effective-mass formalism including the SOI at impurities and $[47,48]$ for that in heterostructures) and describe the motion along the $z$ direction by the pseudospin degree of freedom, that is, by a linear combination of $|L\rangle$ and $|R\rangle$, the lowest-energy state in the left well and that in the right, respectively (Fig. 1).

Our Hamiltonian is

$$
H=H_{\mathrm{W}}+e \boldsymbol{E} \cdot \boldsymbol{r}+V_{\mathrm{imp}}(\boldsymbol{r})+H_{\mathrm{imp}}^{\mathrm{so}},
$$

with

$$
\begin{aligned}
H_{\mathrm{W}} & =\frac{\hat{p}_{x}^{2}+\hat{p}_{y}^{2}}{2 m}+H_{\perp}, \quad H_{\perp}=\frac{\hat{p}_{z}^{2}}{2 m}+V_{\mathrm{well}}(z), \\
H_{\mathrm{imp}}^{\mathrm{so}} & =-\hbar^{-1} \eta \hat{\boldsymbol{\sigma}} \cdot\left(\nabla V_{\mathrm{imp}} \times \hat{\boldsymbol{p}}\right),
\end{aligned}
$$

where $\hat{\boldsymbol{p}}$ is the momentum operator, $\hat{\boldsymbol{p}}=\left(\hat{p}_{x}, \hat{p}_{y}, \hat{p}_{z}\right)=$ $-i \hbar \nabla=-i \hbar\left(\nabla_{x}, \nabla_{y}, \nabla_{z}\right), \hat{\boldsymbol{\sigma}}$ is the Pauli spin operator, $\hat{\boldsymbol{\sigma}}=$ $\left(\hat{\sigma}_{x}, \hat{\sigma}_{y}, \hat{\sigma}_{z}\right), m$ is the effective mass of the conduction band, and $\eta$ is the effective coupling constant of the SOI for an electron in the conduction band. $H_{\perp}$ describes the motion perpendicular to the well with the potential $V_{\text {well }}(z)$, which is induced by the band offset between the well and barrier materials, the gate voltage, the dopant charge distribution, and the electron-electron interaction in the Hartree approximation. $\boldsymbol{E}=\left(E_{x}, E_{y}, 0\right)$ is the in-plane electric field, $e>0$ is the absolute value of the electronic charge, and $V_{\mathrm{imp}}(\boldsymbol{r})$, with $\boldsymbol{r}=(x, y, z)$, is the potential due to impurities, We consider the 
SOI induced by $V_{\text {imp }}(\boldsymbol{r}), H_{\text {imp }}^{\text {so }}$, while we neglect the SOI due to $V_{\text {well }}(z)$ [49-51] and the Dresselhaus SOI [42,52] because we focus on the extrinsic spin Hall effect.

We assume that $V_{\mathrm{imp}}(\boldsymbol{r})$ is the sum of the potentials of each impurity:

$$
V_{\mathrm{imp}}(\boldsymbol{r})=\sum_{i=1}^{N} u_{i}\left(\boldsymbol{r}-\boldsymbol{r}_{i}\right),
$$

where $N$ is the number of impurities and $\boldsymbol{r}_{i}=\left(x_{i}, y_{i}, z_{i}\right)$ is the position vector of the $i$ th impurity. In addition we assume that the potential of the $i$ th impurity $u_{i}$ is attractive for $z_{i}$ in the left well but repulsive for $z_{i}$ in the right well:

$$
\begin{aligned}
& u_{i}\left(\boldsymbol{r}-\boldsymbol{r}_{i}\right)=-u\left(\boldsymbol{r}-\boldsymbol{r}_{i}\right)<0\left(z_{i} \text { in } L\right), \\
& u_{i}\left(\boldsymbol{r}-\boldsymbol{r}_{i}\right)=u\left(\boldsymbol{r}-\boldsymbol{r}_{i}\right)>0\left(z_{i} \text { in } R\right) .
\end{aligned}
$$

We here neglect interlayer matrix elements of $u_{i}\left(\boldsymbol{r}-\boldsymbol{r}_{i}\right)(i=$ $1, \ldots, N)$, that is,

$u_{i}\left(\boldsymbol{r}-\boldsymbol{r}_{i}\right)=|L\rangle\left\langle L\left|u_{i}\left(\boldsymbol{r}-\boldsymbol{r}_{i}\right)\right| L\right\rangle\langle L|+| R\rangle\left\langle R\left|u_{i}\left(\boldsymbol{r}-\boldsymbol{r}_{i}\right)\right| R\right\rangle\langle R|$,

which is satisfied when $u_{i}\left(\boldsymbol{r}-\boldsymbol{r}_{i}\right)=0(i=1, \ldots, N)$ in the barrier region where $\langle R \mid z\rangle \times\langle z \mid L\rangle$ is non-negligible. We also use later, for $i=1, \ldots, N$,

$$
\left\langle L\left|u_{i}\left(\boldsymbol{r}-\boldsymbol{r}_{i}\right)\right| L\right\rangle\left\langle R\left|u_{i}\left(\boldsymbol{r}-\boldsymbol{r}_{i}\right)\right| R\right\rangle=0 .
$$

We introduce the Pauli operators for pseudospin $\hat{\tau}_{\gamma}(\gamma=$ 1,2 , and 3 ) and the identity operator $\hat{\tau}_{0}$, defined by

$$
\begin{aligned}
& \hat{\tau}_{0}=|L\rangle\langle L|+| R\rangle\langle R|, \\
& \hat{\tau}_{1}=|R\rangle\langle L|+| L\rangle\langle R|, \\
& \hat{\tau}_{2}=i|R\rangle\langle L|-i| L\rangle\langle R|, \\
& \hat{\tau}_{3}=|L\rangle\langle L|-| R\rangle\langle R|,
\end{aligned}
$$

in terms of which we express $H_{\perp}$ by

$$
H_{\perp}=-\frac{\hbar \omega}{2} \hat{\tau}_{1}+\frac{\hbar \omega_{V}}{2} \hat{\tau}_{3},
$$

where $\omega=\Delta_{\mathrm{SAS}} / \hbar$ and $\omega_{V}$ represent the interlayer tunneling and the potential offset between two wells, respectively. Here we choose $\omega>0$ so that $(|L\rangle+|R\rangle) / \sqrt{2}$ is the ground state at $\omega_{V}=0$. The ground- and excited-state vectors are denoted by $|G\rangle$ and $|E\rangle$, respectively:

$$
H_{\perp}|n\rangle=\varepsilon_{n}|n\rangle \quad(n=G, E),
$$

where

$$
\varepsilon_{G}=-\frac{1}{2} \hbar \omega_{1}, \quad \varepsilon_{E}=\frac{1}{2} \hbar \omega_{1},
$$

with

$$
\omega_{1}=\sqrt{\omega^{2}+\omega_{V}^{2}} .
$$

Eigenvectors of $H_{\mathrm{W}}$ are constructed with use of $|n\rangle(n=$ $G, E)$ :

$$
H_{\mathrm{W}}|n \boldsymbol{k} \sigma\rangle=\varepsilon_{n k}|n \boldsymbol{k} \sigma\rangle,
$$

where $\boldsymbol{k}=\left(k_{x}, k_{y}\right), k=\sqrt{k_{x}^{2}+k_{y}^{2}}$,

$$
\begin{aligned}
& \hat{p}_{x}|\boldsymbol{k}\rangle=\hbar k_{x}|\boldsymbol{k}\rangle, \quad \hat{p}_{y}|\boldsymbol{k}\rangle=\hbar k_{y}|\boldsymbol{k}\rangle, \\
& \hat{\sigma}_{z}|\sigma\rangle=\sigma|\sigma\rangle, \quad \sigma= \pm 1(\sigma=\uparrow, \downarrow), \\
& \varepsilon_{n k}=\varepsilon_{n}+\varepsilon_{k}, \quad \varepsilon_{k}=\frac{\hbar^{2} k^{2}}{2 m} .
\end{aligned}
$$

\section{ASSUMPTIONS AND APPROXIMATIONS}

We calculate the spin Hall current in the first order of the SOI. Therefore, we neglect spin-flip terms with $\hat{\sigma}_{x}$ and $\hat{\sigma}_{y}$ of $H_{\mathrm{imp}}^{\mathrm{so}}$ in Eq. (1), which give contributions of, at least, the second order in the SOI to the statistical average of the physical quantity $\hat{A}, \operatorname{tr}(\hat{\rho} \hat{A})$, with the density operator $\hat{\rho}$ [53].

The extrinsic spin Hall effect has two contributions, the skew scattering [36-38] and the side jump [54-57] (see $[46,58]$ for a review). Since in this paper we consider samples with long momentum relaxation time in which the skewscattering contribution is dominant [12], we neglect the sidejump contribution.

In addition to the absence of interlayer scattering, $\left\langle R \boldsymbol{k}^{\prime}\left|V_{\text {imp }}\right| L \boldsymbol{k}\right\rangle=0$, derived from Eq. (5), we assume the absence of the interlayer tunneling described by $\Delta_{\text {SAS }}$ within the collision term of the Boltzmann equation; that is, we neglect the combined action of $\Delta_{\mathrm{SAS}}$ and $V_{\text {imp. }}$. These two assumptions on pseudospin, which correspond to those on spin employed in the Dyakonov-Perel spin-relaxation theory [40-42], simplify our problem in that the interlayer transition occurs by the action of $\Delta_{\mathrm{SAS}}$ alone. The effect of $\omega=\Delta_{\mathrm{SAS}} / \hbar$ is negligible during the collision if $\omega \tau_{\text {coll }} \ll 1$, with $\tau_{\text {coll }}$ being the collision time. Since we investigate the crossover region of $\omega \tau_{p} \sim 1, \omega \tau_{\text {coll }} \ll 1$ is satisfied in the case of $\tau_{\text {coll }} \ll \tau_{p}$. The condition $\tau_{\text {coll }} \ll \tau_{p}$ can be restated as having a potential range of each impurity and a Fermi wavelength that are both much shorter than $v_{F} \tau_{p}$, with $v_{F}$ being the Fermi velocity.

We restrict our discussion to the transport which does not depend on individual configurations of impurities randomly distributed in the $x y$ plane. Therefore, we take the average over in-plane configurations of each product of $\left\langle\ell \boldsymbol{k}^{\prime}\left|V_{\text {imp }}\right| \ell \boldsymbol{k}\right\rangle(\ell=$ $L, R)$, which is defined, for $A\left(x_{1}, y_{1}, \ldots, x_{N}, y_{N}\right)$, by

$$
\bar{A} \equiv \frac{1}{S} \int_{S} \cdots \frac{1}{S} \int_{S} A d x_{1} d y_{1} \cdots d x_{N} d y_{N},
$$

where $S$ is the area of the bilayer. Then, in the first order of $V_{\text {imp }}$, we obtain

$$
\overline{\left\langle\ell \boldsymbol{k}^{\prime}\left|V_{\text {imp }}\right| \ell \boldsymbol{k}\right\rangle}=0\left(\boldsymbol{k}^{\prime} \neq \boldsymbol{k}\right) .
$$

On the other hand, when $\boldsymbol{k}^{\prime}=\boldsymbol{k}$, we assume that

$$
\left\langle\ell \boldsymbol{k}\left|V_{\text {imp }}\right| \ell \boldsymbol{k}\right\rangle=0,
$$

which is always satisfied by subtracting the potential $V_{0}(z) \equiv$ $S^{-1} \int_{S} V_{\text {imp }}(\boldsymbol{r}) d x d y$ from $V_{\text {imp }}(\boldsymbol{r})$ while adding $V_{0}(z)$ to $V_{\text {well }}(z)$ in order to keep the Hamiltonian unchanged. Therefore, all terms of the first order in $V_{\text {imp }}$ vanish. In the second order of $V_{\text {imp }}$, we have

$$
\overline{\left\langle\ell^{\prime} \boldsymbol{k}^{\prime \prime}\left|V_{\mathrm{imp}}\right| \ell^{\prime} \boldsymbol{k}^{\prime}\right\rangle\left\langle\ell \boldsymbol{k}^{\prime}\left|V_{\mathrm{imp}}\right| \ell \boldsymbol{k}\right\rangle} \neq 0
$$


only when $\ell^{\prime}=\ell$ and $\boldsymbol{k}^{\prime \prime}=\boldsymbol{k}$, where we used Eq. (6). We additionally assume that the averaged impurity distribution has the inversion symmetry with respect to the plane between the $L$ and $R$ wells so that

$$
\overline{\left\langle L \boldsymbol{k}\left|V_{\mathrm{imp}}\right| L \boldsymbol{k}^{\prime}\right\rangle\left\langle L \boldsymbol{k}^{\prime}\left|V_{\mathrm{imp}}\right| L \boldsymbol{k}\right\rangle}=\overline{\left\langle R \boldsymbol{k}\left|V_{\mathrm{imp}}\right| R \boldsymbol{k}^{\prime}\right\rangle\left\langle R \boldsymbol{k}^{\prime}\left|V_{\mathrm{imp}}\right| R \boldsymbol{k}\right\rangle} \text {. }
$$

Similarly, in the third order of $V_{\text {imp }}$, we have

$$
\overline{\left\langle\ell^{\prime \prime} \boldsymbol{k}^{\prime \prime \prime}\left|V_{\mathrm{imp}}\right| \ell^{\prime \prime} \boldsymbol{k}^{\prime \prime}\right\rangle\left\langle\ell^{\prime} \boldsymbol{k}^{\prime \prime}\left|V_{\mathrm{imp}}\right| \ell^{\prime} \boldsymbol{k}^{\prime}\right\rangle\left\langle\ell \boldsymbol{k}^{\prime}\left|V_{\mathrm{imp}}\right| \ell \boldsymbol{k}\right\rangle} \neq 0
$$

only when $\ell^{\prime \prime}=\ell^{\prime}=\ell$ and $\boldsymbol{k}^{\prime \prime \prime}=\boldsymbol{k}$.

\section{BOLTZMANN EQUATION}

In this paper we derive the formula for the spin Hall current by solving the Boltzmann equation for the density operator $\hat{\rho}$ [53]. Since off-diagonal matrix elements $\left\langle n^{\prime} \boldsymbol{k} \sigma|\hat{\rho}| n \boldsymbol{k} \sigma\right\rangle$ $\left(n^{\prime} \neq n\right)$ are nonzero in the presence of the phase coherence in the pseudospin degree of freedom, it is convenient to introduce an operator in the pseudospin space,

$$
\hat{\rho}_{\boldsymbol{k} \sigma}=\langle\boldsymbol{k} \sigma|\hat{\rho}| \boldsymbol{k} \sigma\rangle .
$$

We call $\hat{\rho}_{\boldsymbol{k} \sigma}$ the distribution operator because it is reminiscent of the distribution function for a state with wave vector $\boldsymbol{k}$ and spin $\sigma$. In uniform steady states, the distribution operator $\hat{\rho}_{\boldsymbol{k} \sigma}$ satisfies the following Boltzmann equation:

$$
\frac{\boldsymbol{F}}{\hbar} \cdot \frac{\partial \hat{\rho}_{\boldsymbol{k} \sigma}}{\partial \boldsymbol{k}}=\left(\frac{\partial \hat{\rho}_{\boldsymbol{k} \sigma}}{\partial t}\right)_{\mathrm{p}+\mathrm{c}}+\left(\frac{\partial \hat{\rho}_{\boldsymbol{k} \sigma}}{\partial t}\right)_{\mathrm{d}},
$$

with $\boldsymbol{F}=(-e) \boldsymbol{E}$. On the left-hand side of this equation, the force term is expressed by the derivative of the operator $\hat{\rho}_{\boldsymbol{k} \sigma}$ with respect to $\boldsymbol{k}$ [59]. On the right-hand side, the first term describes the precession of pseudospin and the collision with impurities, while the second describes the dephasing of pseudospin.

To derive the first term on the right-hand side of Eq. (21), we start with

$$
\frac{d \hat{\rho}}{d t}=\frac{1}{i \hbar}[H, \hat{\rho}]
$$

where the term $e \boldsymbol{E} \cdot \boldsymbol{r}$ is removed from $H$. We divide $H$ into two parts,

$$
H=H_{\mathrm{W}}+V, \quad V=V_{\mathrm{imp}}(\boldsymbol{r})+H_{\mathrm{imp}}^{\mathrm{so}},
$$

and employ the interaction representation:

$$
\hat{\rho}_{I}(t)=U^{\dagger}(t) \hat{\rho}(t) U(t), \quad V_{I}(t)=U^{\dagger}(t) V(t) U(t),
$$

with

$$
U(t)=\exp \left(\frac{1}{i \hbar} H_{\mathrm{W}} t\right)
$$

to obtain, from Eq. (22),

$$
\frac{d \hat{\rho}_{I}}{d t}=\frac{1}{i \hbar}\left[V_{I}, \hat{\rho}_{I}\right]
$$

We turn on the interaction $V$ at $t_{0}$ and solve for the density operator $\hat{\rho}$ at $t$ after a long enough time interval $t-t_{0}$. By integrating both sides of Eq. (26) from $t_{0}$ to $t$, we have
$\hat{\rho}_{I}(t)=\hat{\rho}_{I}\left(t_{0}\right)+(i \hbar)^{-1} \int_{t_{0}}^{t} d t^{\prime}\left[V_{I}\left(t^{\prime}\right), \hat{\rho}_{I}\left(t^{\prime}\right)\right]$. Substituting this into Eq. (26), we obtain, up to third order in $V$,

$$
\frac{d \hat{\rho}_{I}}{d t}=J_{1}(t)+J_{2}(t)+J_{3}(t)
$$

with

$$
\begin{aligned}
& J_{1}(t)=\frac{1}{i \hbar}\left[V_{I}(t), \hat{\rho}_{I}\left(t_{0}\right)\right], \\
& J_{2}(t)=\frac{1}{(i \hbar)^{2}} \int_{t_{0}}^{t} d t^{\prime} P\left[V_{I}(t),\left[V_{I}\left(t^{\prime}\right), \hat{\rho}_{I}\left(t_{0}\right)\right]\right], \\
& J_{3}(t)=\frac{1}{(i \hbar)^{3}} \int_{t_{0}}^{t} d t^{\prime} \int_{t_{0}}^{t^{\prime}} d t^{\prime \prime}\left[V_{I}(t),\left[V_{I}\left(t^{\prime}\right),\left[V_{I}\left(t^{\prime \prime}\right), \hat{\rho}_{I}\left(t_{0}\right)\right]\right]\right],
\end{aligned}
$$

where we replace $\hat{\rho}_{I}\left(t_{0}\right)$ with $\hat{\rho}_{I}(t)$ by considering that the relaxation time of the distribution operator $\tau_{p}$ is much longer than the collision time $\tau_{\text {coll }}$. Writing $\hat{\rho}_{I}(t)$ on the left-hand side of Eq. (27) in terms of $\hat{\rho}(t)$, we obtain

$$
\frac{d \hat{\rho}}{d t}=\frac{1}{i \hbar}\left[H_{\mathrm{W}}, \hat{\rho}\right]+\tilde{J}_{1}(t)+\tilde{J}_{2}(t)+\tilde{J}_{3}(t),
$$

with

$$
\tilde{J}_{n}(t)=U(t) J_{n}(t) U^{\dagger}(t) \quad(n=1,2,3) .
$$

The first term on the right-hand side of Eq. (29) describes the pseudospin precession, while $\tilde{J}_{1}(t), \tilde{J}_{2}(t)$, and $\tilde{J}_{3}(t)$ are due to the impurity scattering.

Since, for $\ell=L, R$,

$$
\left\langle\ell \boldsymbol{k}^{\prime} \sigma|V| \ell \boldsymbol{k} \sigma\right\rangle=\left[1-i \eta \sigma\left(\boldsymbol{k}^{\prime} \times \boldsymbol{k}\right)_{z}\right]\left\langle\ell \boldsymbol{k}^{\prime}\left|V_{\mathrm{imp}}\right| \ell \boldsymbol{k}\right\rangle,
$$

with $\left(\boldsymbol{k}^{\prime} \times \boldsymbol{k}\right)_{z} \equiv k_{x}^{\prime} k_{y}-k_{y}^{\prime} k_{x}$, each matrix element of $J_{n}(t)$ is expressed by products of $\left\langle\ell \boldsymbol{k}^{\prime}\left|V_{\text {imp }}\right| \ell \boldsymbol{k}\right\rangle$, which are replaced by the average over in-plane impurity configurations according to Eq. (14) to obtain the averaged Boltzmann equation for the distribution operator.

We describe the dephasing of pseudospin according to the widely used dephasing model for a two-level system [60] with a constant dephasing time $\tau_{\mathrm{ps}}$ :

$$
\left(\frac{\partial \hat{\rho}_{\boldsymbol{k} \sigma}}{\partial t}\right)_{\mathrm{d}}=-\frac{1}{\tau_{\mathrm{ps}}}\left(|G\rangle\left\langle G\left|\hat{\rho}_{\boldsymbol{k} \sigma}\right| E\right\rangle\langle E|+| E\rangle\left\langle E\left|\hat{\rho}_{\boldsymbol{k} \sigma}\right| G\right\rangle\langle G|\right) .
$$

Here the projection operators $|G\rangle\langle G|$ and $|E\rangle\langle E|$ are expressed by $H_{\perp}$ :

$$
|G\rangle\left\langle G\left|=\frac{1}{2}-\frac{H_{\perp}}{\hbar \omega_{1}}, \quad\right| E\right\rangle\langle E|=\frac{1}{2}+\frac{H_{\perp}}{\hbar \omega_{1}} .
$$

By substituting Eqs. (29) and (32) into Eq. (21), the Boltzmann equation for the distribution operator becomes

$$
\frac{\boldsymbol{F}}{\hbar} \cdot \frac{\partial \hat{\rho}_{\boldsymbol{k} \sigma}}{\partial \boldsymbol{k}}=\frac{1}{i \hbar}\left[H_{\perp}, \hat{\rho}_{\boldsymbol{k} \sigma}\right]+\hat{C}^{(2)}+\hat{C}^{(3)}+\left(\frac{\partial \hat{\rho}_{\boldsymbol{k} \sigma}}{\partial t}\right)_{\mathrm{d}},
$$

with

$$
\begin{array}{r}
\hat{C}^{(2)}=\sum_{\boldsymbol{k}^{\prime}} W_{\boldsymbol{k}^{\prime} \boldsymbol{k}}^{(2)}\left(-\hat{\rho}_{\boldsymbol{k} \sigma}+|L\rangle \rho_{\boldsymbol{k}^{\prime} \sigma}^{L L}\langle L|+| R\rangle \rho_{\boldsymbol{k}^{\prime} \sigma}^{R R}\langle R|\right), \\
\hat{C}^{(3)}=\sum_{\boldsymbol{k}^{\prime}} W_{\boldsymbol{k} \boldsymbol{k}^{\prime} \sigma}^{(3) \mathrm{ss}}\left(|L\rangle \rho_{\boldsymbol{k}^{\prime} \sigma}^{L L}\langle L|-| R\rangle \rho_{\boldsymbol{k}^{\prime} \sigma}^{R R}\langle R|\right),
\end{array}
$$


and $\rho_{k \sigma}^{L L}=\left\langle L\left|\hat{\rho}_{\boldsymbol{k} \sigma}\right| L\right\rangle$. The transition rate in the second order of $V, W_{\boldsymbol{k}^{\prime} \boldsymbol{k}}^{(2)}$, is defined by

$$
W_{\boldsymbol{k}^{\prime} \boldsymbol{k}}^{(2)} \equiv W_{L \boldsymbol{k}^{\prime} L \boldsymbol{k}}^{(2)}=W_{R \boldsymbol{k}^{\prime} R \boldsymbol{k}}^{(2)},
$$

with

$$
W_{L k^{\prime} L \boldsymbol{k}}^{(2)}=\frac{2 \pi}{\hbar} \delta\left(\varepsilon_{k^{\prime}}-\varepsilon_{k}\right) \overline{\left|\left\langle L \boldsymbol{k}^{\prime}\left|V_{\mathrm{imp}}\right| L \boldsymbol{k}\right\rangle\right|^{2}},
$$

and gives the momentum relaxation. On the other hand, the third-order transition rate $W_{\boldsymbol{k} \boldsymbol{k}^{\prime} \sigma}^{(3) \mathrm{ss}}$ is defined by

$$
W_{\boldsymbol{k} \boldsymbol{k}^{\prime} \sigma}^{(3) \mathrm{ss}} \equiv W_{L \boldsymbol{k} L \boldsymbol{k}^{\prime}}^{\sigma(3) \mathrm{ss}}=-W_{R \boldsymbol{k} \boldsymbol{k} \boldsymbol{k}^{\prime}}^{\sigma(3) \mathrm{ss}},
$$

where

$$
W_{L k L k^{\prime}}^{\sigma(3) s s}=\text { first-order-in- } \eta \text { terms of } W_{L k L k^{\prime}}^{\sigma(3)}
$$

and

$$
W_{L \boldsymbol{k}^{\prime} L \boldsymbol{k}}^{\sigma(3)}=\frac{2 \pi}{\hbar} \delta\left(\varepsilon_{k^{\prime}}-\varepsilon_{k}\right) \sum_{\boldsymbol{k}^{\prime \prime}}\left[\frac{\left(\boldsymbol{L} \boldsymbol{k} \boldsymbol{k}^{\prime} \boldsymbol{k}^{\prime \prime} \boldsymbol{k} \sigma\right)}{\varepsilon_{k}-\varepsilon_{k^{\prime \prime}}+i \delta}+\text { c.c. }\right],
$$

$\left(L \boldsymbol{k} \boldsymbol{k}^{\prime} \boldsymbol{k}^{\prime \prime} \boldsymbol{k} \sigma\right)=\overline{\left\langle L \boldsymbol{k} \sigma|V| L \boldsymbol{k}^{\prime} \sigma\right\rangle\left\langle L \boldsymbol{k}^{\prime} \sigma|V| L \boldsymbol{k}^{\prime \prime} \sigma\right\rangle\left\langle L \boldsymbol{k}^{\prime \prime} \sigma|V| L \boldsymbol{k} \sigma\right\rangle}$.

The third-order transition rates $W_{L k L k^{\prime}}^{\sigma(3) s s}$ and $W_{R k R k^{\prime}}^{\sigma(3) s s}$, which represent the skew scattering in the lowest order, are opposite in sign because the sign of the impurity potential is opposite between $L$ and $R$ [Eq. (4)].

Here we find it convenient to decompose the distribution operator $\hat{\rho}_{\boldsymbol{k} \sigma}$ into a linear combination of the pseudospin operator $\hat{\tau}_{\gamma}$ and to rewrite the Boltzmann equation (34) in terms of the corresponding component, according to the similar decomposition for the spin degree of freedom [61-63],

$$
\hat{\rho}_{\boldsymbol{k} \sigma}=\frac{1}{2} \sum_{\gamma=0}^{3} \tau_{\gamma \boldsymbol{k} \sigma} \hat{\tau}_{\gamma},
$$

where $\tau_{\gamma \boldsymbol{k} \sigma}$, twice the $\gamma$ component of $\hat{\rho}_{\boldsymbol{k} \sigma}$, is given by the statistical average of $\hat{\tau}_{\gamma}$ in a state with momentum $\boldsymbol{k}$ and spin $\sigma$

$$
\tau_{\gamma \boldsymbol{k} \sigma}=\operatorname{tr}_{\tau}\left(\hat{\rho}_{\boldsymbol{k} \sigma} \hat{\tau}_{\gamma}\right) \equiv \sum_{\ell}\left\langle\ell\left|\hat{\rho}_{\boldsymbol{k} \sigma} \hat{\tau}_{\gamma}\right| \ell\right\rangle .
$$

Using $\tau_{\gamma k \sigma}$, the Boltzmann equation is written as follows:

$$
\begin{gathered}
\frac{\boldsymbol{F}}{\hbar} \cdot \frac{\partial \tau_{0 k \sigma}}{\partial \boldsymbol{k}}=\sum_{\boldsymbol{k}^{\prime}}\left[W_{\boldsymbol{k}^{\prime} \boldsymbol{k}}^{(2)}\left(-\tau_{0 k \sigma}+\tau_{0 \boldsymbol{k}^{\prime} \sigma}\right)+W_{\boldsymbol{k} \boldsymbol{k}^{\prime} \sigma}^{(3) \mathrm{ss}} \tau_{3 \boldsymbol{k}^{\prime} \sigma}\right] \\
\frac{\boldsymbol{F}}{\hbar} \cdot \frac{\partial \tau_{1 k \sigma}}{\partial \boldsymbol{k}}=-\omega_{V} \tau_{2 \boldsymbol{k} \sigma}-\tau_{l}^{-1} \tau_{1 \boldsymbol{k} \sigma}+\left(\frac{\partial \tau_{1 \boldsymbol{k} \sigma}}{\partial t}\right)_{\mathrm{d}} \\
\frac{\boldsymbol{F}}{\hbar} \cdot \frac{\partial \tau_{2 \boldsymbol{k} \sigma}}{\partial \boldsymbol{k}}=\omega \tau_{3 \boldsymbol{k} \sigma}+\omega_{V} \tau_{1 k \sigma}-\tau_{l}^{-1} \tau_{2 \boldsymbol{k} \sigma}+\left(\frac{\partial \tau_{2 \boldsymbol{k} \sigma}}{\partial t}\right)_{\mathrm{d}} \\
\frac{\boldsymbol{F}}{\hbar} \cdot \frac{\partial \tau_{3 \boldsymbol{k} \sigma}}{\partial \boldsymbol{k}}=-\omega \tau_{2 \boldsymbol{k} \sigma}+\sum_{\boldsymbol{k}^{\prime}}\left[W_{\boldsymbol{k}^{\prime} \boldsymbol{k}}^{(2)}\left(-\tau_{3 \boldsymbol{k} \sigma}+\tau_{3 \boldsymbol{k}^{\prime} \sigma}\right)\right. \\
\left.+W_{\boldsymbol{k} \boldsymbol{k}^{\prime} \sigma}^{(3) \mathrm{ss}} \tau_{0 \boldsymbol{k}^{\prime} \sigma}\right]+\left(\frac{\partial \tau_{3 \boldsymbol{k} \sigma}}{\partial t}\right)_{\mathrm{d}}
\end{gathered}
$$

where

$$
\tau_{l}^{-1} \equiv \sum_{\boldsymbol{k}^{\prime}} W_{\boldsymbol{k}^{\prime} k}^{(2)}
$$

$$
\begin{aligned}
& \left(\frac{\partial \tau_{1 k \sigma}}{\partial t}\right)_{\mathrm{d}}=-\tau_{\mathrm{ps}}^{-1}\left(s_{\omega}^{2} \tau_{1 k \sigma}+s_{\omega} c_{\omega} \tau_{3 k \sigma}\right), \\
& \left(\frac{\partial \tau_{2 k \sigma}}{\partial t}\right)_{\mathrm{d}}=-\tau_{\mathrm{ps}}^{-1} \tau_{2 k \sigma}, \\
& \left(\frac{\partial \tau_{3 k \sigma}}{\partial t}\right)_{\mathrm{d}}=-\tau_{\mathrm{ps}}^{-1}\left(s_{\omega} c_{\omega} \tau_{1 k \sigma}+c_{\omega}^{2} \tau_{3 k \sigma}\right),
\end{aligned}
$$

with

$$
s_{\omega}=\frac{\omega_{V}}{\omega_{1}}, \quad c_{\omega}=\frac{\omega}{\omega_{1}}, \quad s_{\omega}^{2}+c_{\omega}^{2}=1
$$

\section{DISTRIBUTION OPERATOR}

\section{A. Distribution operator in the absence of the in-plane electric field}

We can choose, as the density operator of the zeroth order in $E=|\boldsymbol{E}|$,

$$
\hat{\rho}^{(0)}=f_{0}\left(H_{\mathrm{W}}\right), \quad f_{0}(\varepsilon)=\left[\exp \left(\frac{\varepsilon-\mu}{k_{\mathrm{B}} T}\right)+1\right]^{-1},
$$

where $f_{0}(\varepsilon)$ is the Fermi-Dirac distribution function with chemical potential $\mu$ and temperature $T$. Since

$$
\hat{\rho}_{\boldsymbol{k} \sigma}^{(0)}=\sum_{n=G, E}|n\rangle f_{0}\left(\varepsilon_{n k}\right)\langle n|,
$$

we obtain $\tau_{\gamma \boldsymbol{k} \sigma}^{(0)}$, the $\gamma$ component of $2 \hat{\rho}_{\boldsymbol{k} \sigma}^{(0)}$, as follows:

$$
\begin{aligned}
\tau_{0 k \sigma}^{(0)} & =f_{0}\left(\varepsilon_{G k}\right)+f_{0}\left(\varepsilon_{E k}\right), \\
\tau_{1 k \sigma}^{(0)} & =c_{\omega}\left[f_{0}\left(\varepsilon_{G k}\right)-f_{0}\left(\varepsilon_{E k}\right)\right], \\
\tau_{2 k \sigma}^{(0)} & =0, \\
\tau_{3 k \sigma}^{(0)} & =s_{\omega}\left[-f_{0}\left(\varepsilon_{G k}\right)+f_{0}\left(\varepsilon_{E k}\right)\right] .
\end{aligned}
$$

\section{B. Distribution operator in the linear response to the in-plane electric field}

The Boltzmann equation in the first order of $E$ is obtained by substituting $\tau_{\gamma k \sigma}^{(0)}$ into the force term and $\tau_{\gamma k \sigma}^{(1)}$ into other terms. We seek the solution for $\tau_{\gamma \boldsymbol{k} \sigma}^{(1)}$ of the form

$$
\tau_{\gamma \boldsymbol{k} \sigma}^{(1)}=\boldsymbol{a}_{\gamma k \sigma} \cdot \hbar \boldsymbol{k} .
$$

Then the Boltzmann equation gives the following coupled equations for $\boldsymbol{a}_{\gamma k \sigma}$ :

$$
\begin{aligned}
\frac{\boldsymbol{F}}{m}\left(f_{G}^{\prime}+f_{E}^{\prime}\right)= & -\tau_{p}^{-1} \boldsymbol{a}_{0 k \sigma}+\tau_{\mathrm{ss}}^{-1} \sigma\left(\boldsymbol{e}_{z} \times \boldsymbol{a}_{3 k \sigma}\right), \\
\frac{\boldsymbol{F}}{m} c_{\omega}\left(f_{G}^{\prime}-f_{E}^{\prime}\right)= & -\omega_{V} \boldsymbol{a}_{2 k \sigma}-\tau_{l}^{-1} \boldsymbol{a}_{1 k \sigma} \\
& -\tau_{\mathrm{ps}}^{-1}\left(s_{\omega}^{2} \boldsymbol{a}_{1 k \sigma}+s_{\omega} c_{\omega} \boldsymbol{a}_{3 k \sigma}\right), \\
0= & \omega \boldsymbol{a}_{3 k \sigma}+\omega_{V} \boldsymbol{a}_{1 k \sigma}-\left(\tau_{l}^{-1}+\tau_{\mathrm{ps}}^{-1}\right) \boldsymbol{a}_{2 k \sigma}, \\
\frac{\boldsymbol{F}}{m} s_{\omega}\left(-f_{G}^{\prime}+f_{E}^{\prime}\right)= & -\omega \boldsymbol{a}_{2 k \sigma}-\tau_{p}^{-1} \boldsymbol{a}_{3 k \sigma}+\tau_{\mathrm{ss}}^{-1} \sigma\left(\boldsymbol{e}_{z} \times \boldsymbol{a}_{0 k \sigma}\right) \\
& -\tau_{\mathrm{ps}}^{-1}\left(s_{\omega} c_{\omega} \boldsymbol{a}_{1 k \sigma}+c_{\omega}^{2} \boldsymbol{a}_{3 k \sigma}\right),
\end{aligned}
$$


with

$$
\begin{aligned}
\boldsymbol{e}_{z} \times \boldsymbol{a} & \equiv\left(-a_{y}, a_{x}\right), \quad \boldsymbol{a}=\left(a_{x}, a_{y}\right), \\
f_{n}^{\prime} & \equiv \frac{\partial f_{0}}{\partial \varepsilon}\left(\varepsilon_{n k}\right), \\
\tau_{p}^{-1} & \equiv \sum_{\boldsymbol{k}^{\prime}} W_{\boldsymbol{k}^{\prime} \boldsymbol{k}}^{(2)}(1-\cos \theta), \\
\tau_{\mathrm{ss}}^{-1} & \equiv \sum_{\boldsymbol{k}^{\prime}} W_{\boldsymbol{k}^{\prime} \boldsymbol{k} \uparrow}^{(3) \mathrm{ss}} \sin \theta,
\end{aligned}
$$

where $\theta$ is the angle of $\boldsymbol{k}^{\prime}$ relative to that of $\boldsymbol{k}$.

We divide $\boldsymbol{a}_{\gamma k \sigma}$ into zeroth-order and first-order terms with respect to the SOI:

$$
\boldsymbol{a}_{\gamma k \sigma}=\boldsymbol{a}_{\gamma k \sigma}^{[0]}+\boldsymbol{a}_{\gamma k \sigma}^{[1]} .
$$

Then the solution for $\boldsymbol{a}_{\gamma k \sigma}^{[0]}$ and $\boldsymbol{a}_{\gamma k \sigma}^{[1]}(\gamma=0,3)$ is found to be

$$
\begin{aligned}
\boldsymbol{a}_{0 k \sigma}^{[0]} & =\left(-f_{G}^{\prime}-f_{E}^{\prime}\right) \frac{\tau_{p} \boldsymbol{F}}{m}, \\
\boldsymbol{a}_{3 k \sigma}^{[0]} & =\frac{\left(\gamma_{k}+1\right) s_{\omega}}{\gamma_{k} \tau_{p} \tau_{l}^{-1}+1}\left(f_{G}^{\prime}-f_{E}^{\prime}\right) \frac{\tau_{p} \boldsymbol{F}}{m}, \\
\boldsymbol{a}_{0 k \sigma}^{[1]} & =g_{k}\left(\gamma_{k}+1\right) s_{\omega}\left(f_{G}^{\prime}-f_{E}^{\prime}\right) \sigma\left(\boldsymbol{e}_{z} \times \boldsymbol{F}\right), \\
\boldsymbol{a}_{3 k \sigma}^{[1]} & =g_{k}\left(-f_{G}^{\prime}-f_{E}^{\prime}\right) \sigma\left(\boldsymbol{e}_{z} \times \boldsymbol{F}\right),
\end{aligned}
$$

where

$$
g_{k}=\frac{1}{\gamma_{k} \tau_{p} \tau_{l}^{-1}+1} \frac{\tau_{p}^{2}}{m \tau_{\mathrm{ss}}}, \quad \gamma_{k}=\frac{\omega^{2}+v_{l \varphi} \tau_{\mathrm{ps}}^{-1} c_{\omega}^{2}}{\omega_{V}^{2}+v_{l \varphi}^{s} \nu_{l \varphi}},
$$

with

$$
v_{l \varphi}=\tau_{l}^{-1}+\tau_{\mathrm{ps}}^{-1}, \quad \nu_{l \varphi}^{s}=\tau_{l}^{-1}+\tau_{\mathrm{ps}}^{-1} s_{\omega}^{2},
$$

while that for $\gamma=1,2$ is obtained from

$$
\begin{aligned}
& \boldsymbol{a}_{1 k \sigma}=\frac{\alpha_{1}}{\beta_{0}} \boldsymbol{a}_{3 k \sigma}-\frac{\tau_{p}^{-1} v_{l \varphi} c_{\omega}}{\beta_{0}}\left(f_{G}^{\prime}-f_{E}^{\prime}\right) \frac{\tau_{p} \boldsymbol{F}}{m}, \\
& \boldsymbol{a}_{2 k \sigma}=\frac{\alpha_{2}}{\beta_{0}} \boldsymbol{a}_{3 k \sigma}-\frac{\tau_{p}^{-1} \omega_{V} c_{\omega}}{\beta_{0}}\left(f_{G}^{\prime}-f_{E}^{\prime}\right) \frac{\tau_{p} \boldsymbol{F}}{m},
\end{aligned}
$$

with

$$
\begin{aligned}
& \alpha_{1}=-v_{l \varphi} \tau_{\mathrm{ps}}^{-1} s_{\omega} c_{\omega}-\omega_{V} \omega, \\
& \alpha_{2}=\omega v_{l \varphi}^{s}-\omega_{V} \tau_{\mathrm{ps}}^{-1} s_{\omega} c_{\omega}, \\
& \beta_{0}=v_{l \varphi} v_{l \varphi}^{s}+\omega_{V}^{2} .
\end{aligned}
$$

\section{SPIN HALL CURRENT}

\section{A. Spin Hall conductivity}

Here we introduce the number current density (electron flux density) carrying the $z$ component of spin and the $\gamma$ component of pseudospin in the direction $\mu=x, y$, which is defined by

$$
j_{\mu}^{\mathrm{s} \gamma} \equiv \frac{1}{2 S} \operatorname{tr}\left(\hat{\rho} \hat{\sigma}_{z} \hat{\tau}_{\gamma} \frac{\hat{p}_{\mu}}{m}\right)=\frac{1}{2 S} \sum_{k \sigma} \sigma \tau_{\gamma k \sigma} \frac{\hbar k_{\mu}}{m} .
$$

Such spin-pseudospin current, $\boldsymbol{j}^{\mathrm{s} \gamma}=\left(j_{x}^{\mathrm{s} \gamma}, j_{y}^{\mathrm{s} \gamma}\right)$, is given in the first order of the SOI by

$$
\boldsymbol{j}^{\mathrm{s} \gamma}=\frac{1}{2 S} \sum_{\boldsymbol{k}} \varepsilon_{k}\left(\boldsymbol{a}_{\gamma k \uparrow}^{[1]}-\boldsymbol{a}_{\gamma k \downarrow}^{[1]}\right)=\frac{1}{S} \sum_{\boldsymbol{k}} \varepsilon_{k} \boldsymbol{a}_{\gamma k \uparrow}^{[1]} .
$$

We define the spin Hall conductivity in the $\gamma$ component $\sigma_{y x}^{\mathrm{s} \gamma}$ by

$$
j_{y}^{\mathrm{s} \gamma}=\sigma_{y x}^{\mathrm{s} \gamma} E_{x} .
$$

Substituting the formula of $\boldsymbol{a}_{\gamma k \sigma}^{[1]}$ in Eqs. (54) and (57) into Eq. (60), we obtain the following formula for the spin Hall conductivity:

$$
\begin{aligned}
& \sigma_{y x}^{\mathrm{s} 0}=-\frac{e}{S} \sum_{k} \varepsilon_{k} g_{k}\left(\gamma_{k}+1\right) s_{\omega}\left(f_{G}^{\prime}-f_{E}^{\prime}\right), \\
& \sigma_{y x}^{\mathrm{s} 1}=-\frac{e}{S} \sum_{k} \varepsilon_{k} g_{k} \frac{\alpha_{1}}{\beta_{0}}\left(-f_{G}^{\prime}-f_{E}^{\prime}\right), \\
& \sigma_{y x}^{\mathrm{s} 2}=-\frac{e}{S} \sum_{k} \varepsilon_{k} g_{k} \frac{\alpha_{2}}{\beta_{0}}\left(-f_{G}^{\prime}-f_{E}^{\prime}\right), \\
& \sigma_{y x}^{\mathrm{s} 3}=-\frac{e}{S} \sum_{k} \varepsilon_{k} g_{k}\left(-f_{G}^{\prime}-f_{E}^{\prime}\right) .
\end{aligned}
$$

Here we consider the case where $k_{\mathrm{B}} T \ll \varepsilon_{\mathrm{F}}, \Delta_{\mathrm{SAS}} \ll \varepsilon_{\mathrm{F}}$, and $\hbar \omega_{V} \ll \varepsilon_{\mathrm{F}}$, with $\varepsilon_{\mathrm{F}}=\hbar^{2} k_{\mathrm{F}}^{2} / 2 m$ being the Fermi energy. Then, by replacing $\tau_{p}(\varepsilon), \tau_{l}(\varepsilon)$, and $\tau_{\mathrm{ss}}(\varepsilon)$, which are slowly varying functions of $\varepsilon$, with their value at $\varepsilon_{\mathrm{F}}$, we obtain

$$
\begin{gathered}
\sigma_{y x}^{\mathrm{s} 0}=0, \\
\frac{\sigma_{y x}^{\mathrm{s} 3}}{\sigma_{0}^{\mathrm{sH}}}=\frac{1}{\gamma_{k} \tau_{p} \tau_{l}^{-1}+1},
\end{gathered}
$$

with

$$
\sigma_{0}^{\mathrm{sH}}=-\frac{2 N_{\mathrm{e}} e \tau_{p}^{2}}{m \tau_{\mathrm{ss}}},
$$

where $N_{\mathrm{e}}$ is the areal electron density per spin per layer, $\tau_{l}=\tau_{l}\left(\varepsilon_{\mathrm{F}}\right), \tau_{p}=\tau_{p}\left(\varepsilon_{\mathrm{F}}\right)$, and $\tau_{\mathrm{ss}}=\tau_{\mathrm{ss}}\left(\varepsilon_{\mathrm{F}}\right)$.

When $\omega_{V}=0$, we have $s_{\omega}=0$ and $\gamma_{k}=\omega^{2} \tau_{l} \nu_{l \varphi}^{-1}+\tau_{l} \tau_{\mathrm{ps}}^{-1}$ and obtain

$$
\frac{\sigma_{y x}^{\mathrm{s} 3}}{\sigma_{0}^{\mathrm{sH}}}=\frac{\left(1+\tau_{p} \tau_{\mathrm{ps}}^{-1}\right)^{-1}}{1+\omega^{2}\left[\left(\tau_{p}^{-1}+\tau_{\mathrm{ps}}^{-1}\right)\left(\tau_{l}^{-1}+\tau_{\mathrm{ps}}^{-1}\right)\right]^{-1}} .
$$

When $\tau_{\mathrm{ps}}^{-1}=0$, we have $\gamma_{k}=\omega^{2} /\left(\omega_{V}^{2}+\tau_{l}^{-2}\right)$ and obtain

$$
\frac{\sigma_{y x}^{\mathrm{s3}}}{\sigma_{0}^{\mathrm{sH}}}=\frac{\omega_{V}^{2} \tau_{l}^{2}+1}{1+\left(\omega^{2} \tau_{p} \tau_{l}^{-1}+\omega_{V}^{2}\right) \tau_{l}^{2}} .
$$

When $\omega_{V}=0$ and $\tau_{\mathrm{ps}}^{-1}=0$, we obtain

$$
\frac{\sigma_{y x}^{\mathrm{s} 3}}{\sigma_{0}^{\mathrm{sH}}}=\frac{1}{1+\omega^{2} \tau_{p} \tau_{l}},
$$

which is further simplified to $\tau_{l}=\tau_{p}$ and

$$
\frac{\sigma_{y x}^{\mathrm{s} 3}}{\sigma_{0}^{\mathrm{sH}}}=\frac{1}{1+\omega^{2} \tau_{p}^{2}}
$$



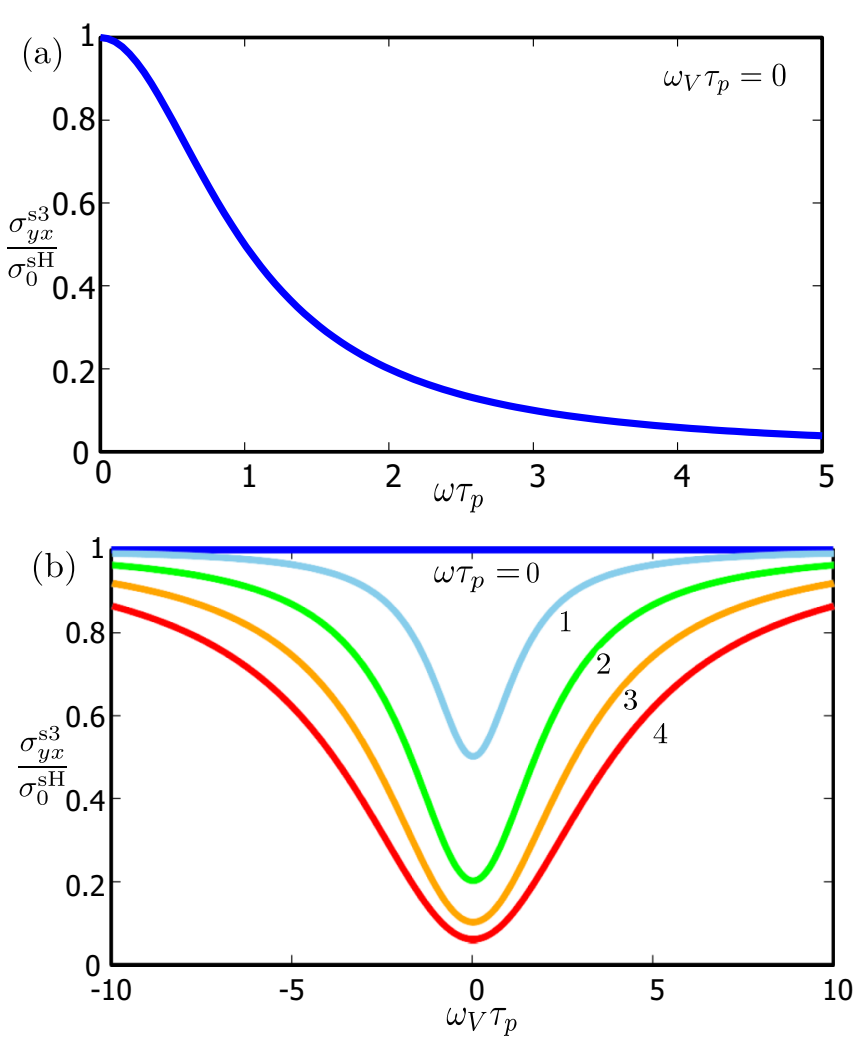

FIG. 2. Normalized antiparallel spin Hall conductivity $\sigma_{y x}^{\mathrm{s} 3} / \sigma_{0}^{\mathrm{sH}}$. (a) The dependence on the pseudospin-precession frequency, $\omega=$ $\Delta_{\text {SAS }} / \hbar$ [Eq. (69)], which shows that the momentum relaxation time $\tau_{p}$ determines the crossover position. (b) The dependence on the potential offset between the $L$ and $R$ layers $\hbar \omega_{V}$ [Eq. (67) at $\tau_{l}=\tau_{p}$ ].

when the potential range of each impurity is much shorter than the Fermi wavelength. Equations (67) and (69) are plotted in Fig. 2.

On the other hand, $\sigma_{y x}^{\mathrm{s} 1}$ and $\sigma_{y x}^{\mathrm{s} 2}$ become nonzero at $\omega \neq 0$ :

$$
\frac{\sigma_{y x}^{\mathrm{s} 1}}{\sigma_{y x}^{\mathrm{s} 3}}=\frac{\alpha_{1}}{\beta_{0}}, \quad \frac{\sigma_{y x}^{\mathrm{s} 2}}{\sigma_{y x}^{\mathrm{s} 3}}=\frac{\alpha_{2}}{\beta_{0}} .
$$

In particular, when $\omega_{V}=0, \tau_{\mathrm{ps}}^{-1}=0$, and $\tau_{l}=\tau_{p}$, we obtain

$$
\sigma_{y x}^{\mathrm{s} 1}=0, \quad \sigma_{y x}^{\mathrm{s} 2}=\omega \tau_{p} \sigma_{y x}^{\mathrm{s} 3},
$$

which leads to an interesting relation:

$$
\sigma_{y x}^{\mathrm{s} 3}(\omega)+i \sigma_{y x}^{\mathrm{s} 2}(\omega)=\frac{\sigma_{0}^{\mathrm{sH}}}{1-i \omega \tau_{p}} .
$$

\section{B. Hanle effect of the pseudospin current}

In this section we show that the reduction by the pseudospin precession $\omega$ of the pseudospin components of the spin Hall conductivity, $\sigma_{y x}^{\mathrm{s} 2}(\omega)$ and $\sigma_{y x}^{\mathrm{s} 3}(\omega)$, in the case where $\omega_{V}=$ $0, k_{\mathrm{B}} T \ll \varepsilon_{\mathrm{F}}$, and $\Delta_{\mathrm{SAS}} \ll \varepsilon_{\mathrm{F}}[$ Eq. (72)] can be regarded as the Hanle effect for the pseudospin current. For this purpose we go back to the Boltzmann equation [Eq. (44)] and take only terms first order in $|\boldsymbol{E}|$ and up to first order in $\eta$, as we have done in Sec. V B. We further neglect the potential offset $\omega_{V}$ as in Eq. (72) and restore the time-derivative term, which actually vanishes in steady states. Then we obtain

$$
\begin{aligned}
\frac{\partial \tau_{0 \boldsymbol{k} \sigma}^{(1)}}{\partial t}+\frac{\boldsymbol{F}}{\hbar} \cdot \frac{\partial \tau_{0 \boldsymbol{k} \sigma}^{(0)}}{\partial \boldsymbol{k}}= & -\tau_{p}^{-1} \tau_{0 \boldsymbol{k} \sigma}^{(1)}, \\
\frac{\partial \tau_{1 \boldsymbol{k} \sigma}^{(1)}}{\partial t}+\frac{\boldsymbol{F}}{\hbar} \cdot \frac{\partial \tau_{1 \boldsymbol{k} \sigma}^{(0)}}{\partial \boldsymbol{k}}= & -\tau_{l}^{-1} \tau_{1 \boldsymbol{k} \sigma}^{(1)}, \\
\frac{\partial \tau_{2 \boldsymbol{k} \sigma}^{(1)}}{\partial t}+\frac{\boldsymbol{F}}{\hbar} \cdot \frac{\partial \tau_{2 \boldsymbol{k} \sigma}^{(0)}}{\partial \boldsymbol{k}}= & =\omega \tau_{3 \boldsymbol{k} \sigma}^{(1)}-\left(\tau_{l}^{-1}+\tau_{\mathrm{ps}}^{-1}\right) \tau_{2 \boldsymbol{k} \sigma}^{(1)}, \\
\frac{\partial \tau_{3 \boldsymbol{k} \sigma}^{(1)}}{\partial t}+\frac{\boldsymbol{F}}{\hbar} \cdot \frac{\partial \tau_{3 \boldsymbol{k} \sigma}^{(0)}}{\partial \boldsymbol{k}}= & -\omega \tau_{2 \boldsymbol{k} \sigma}^{(1)}-\left(\tau_{p}^{-1}+\tau_{\mathrm{ps}}^{-1}\right) \tau_{3 \boldsymbol{k} \sigma}^{(1)} \\
& -\frac{\boldsymbol{F}_{\mathrm{sH}}}{\hbar} \cdot \frac{\partial \tau_{0 \boldsymbol{k} \sigma}^{(0)}}{\partial \boldsymbol{k}},
\end{aligned}
$$

where

$$
\boldsymbol{F}_{\mathrm{sH}}=\tau_{\mathrm{ss}}^{-1} \tau_{p} \sigma\left(\boldsymbol{e}_{z} \times \boldsymbol{F}\right)
$$

can be thought of as a force generating the spin Hall current. We find that $\tau_{\mathrm{ss}}^{-1}$ represents the strength of this force, while $\tau_{p}, \tau_{l}$, and $\tau_{\mathrm{ps}}$ determine the relaxation time for $\tau_{\gamma \boldsymbol{k} \sigma}^{(1)}$.

We derive the equation of motion for $j_{y}^{\mathrm{s} \gamma}$ from Eq. (73). By again employing the approximation made in Sec. VIA, where $\tau_{p}(\varepsilon) \approx \tau_{p}\left(\varepsilon_{\mathrm{F}}\right), \tau_{l}(\varepsilon) \approx \tau_{l}\left(\varepsilon_{\mathrm{F}}\right)$, and $\tau_{\mathrm{ss}}(\varepsilon) \approx \tau_{\mathrm{ss}}\left(\varepsilon_{\mathrm{F}}\right)$, we obtain the following equations:

$$
\begin{aligned}
& \frac{d j_{y}^{\mathrm{s} 0}}{d t}=-\tau_{p}^{-1} j_{y}^{\mathrm{s} 0}, \\
& \frac{d j_{y}^{\mathrm{s} 1}}{d t}=-\tau_{l}^{-1} j_{y}^{\mathrm{s} 1}, \\
& \frac{d j_{y}^{\mathrm{s} 2}}{d t}=\omega j_{y}^{\mathrm{s} 3}-\left(\tau_{l}^{-1}+\tau_{\mathrm{ps}}^{-1}\right) j_{y}^{\mathrm{s} 2}, \\
& \frac{d j_{y}^{\mathrm{s} 3}}{d t}=-\omega j_{y}^{\mathrm{s} 2}-\left(\tau_{p}^{-1}+\tau_{\mathrm{ps}}^{-1}\right) j_{y}^{\mathrm{s} 3}+\tau_{p}^{-1} \sigma_{0}^{\mathrm{sH}} E_{x} .
\end{aligned}
$$

The first equation gives $j_{y}^{\mathrm{s} 0}=0\left(\sigma_{y x}^{\mathrm{s} 0}=0\right)$ in steady states. The rest of the equations describe the dynamics of the pseudospin current. The pseudospin precesses with frequency $\omega$ around "axis 1." The pseudospin current relaxes by the action of the pseudospin dephasing $\tau_{\mathrm{ps}}^{-1}$ and the momentum relaxation, the latter of which is anisotropic with respect to the pseudospin direction: $\tau_{l}$ for the first and second pseudospin components and $\tau_{p}$ for the third. The term proportional to $E_{x}$ describes the generation of the spin Hall current which carries the third component of pseudospin together with the $z$ component of spin. The last three equations in Eq. (75), when simplified by assuming $\tau_{\mathrm{ps}}^{-1}=0$ and $\tau_{l}=\tau_{p}$, reproduce the reduction by $\omega$ of the second and third components [Eq. (72)]. Since the present reduction is found to be based on the equation which is the same in form as that leading to the Hanle effect [45], it can be regarded as the Hanle effect for the pseudospin current.

\section{Possible experiments using the potential offset}

As a possible experiment to observe the antiparallel spin Hall current, we first propose extending (to the present bilayer system) the nonlocal detection in the $\mathrm{H}$-shaped geometry of the spin Hall current which was proposed [64] and experimentally demonstrated [65] in a single quantum well where the applied electric field along the left arm of the 
$\mathrm{H}$ creates the spin Hall current from the left to the right arm and the spin Hall current in turn generates the detected electric field in the right arm. In the present bilayer of the $\mathrm{H}$-shaped geometry, the applied electric field along the left arm creates the antiparallel spin Hall current from the left to the right arm, and the antiparallel spin Hall current in turn generates the detected electric field in the right arm, which shows the different directions between two configurations of the antiparallel spin Hall current, $\uparrow \downarrow$ and $\downarrow \uparrow$, in two layers (the proposed nonlocal detection of the antiparallel spin Hall current has an essential aspect in common with a readout method of the antiferromagnetic memory which was recently demonstrated experimentally [66,67]). The long spin diffusion length necessary for such nonlocal measurement was experimentally realized (see, for example, Refs. [68,69]), and even longer spin diffusion lengths will be made possible by choosing appropriate materials [70].

Another possible method to detect the antiparallel spin Hall current is to use the dynamic nuclear polarization (DNP) which is generated by nonequilibrium electron spin accumulation through the hyperfine interaction. Such a DNP, generated by the current-induced electron spin polarization in an InGaAs epilayer [71], was successfully observed, in addition to that created by spin-polarized electrons injected from a metallic ferromagnet into a GaAs quantum well [72]. In order to detect the electron spin accumulation induced by the antiparallel spin Hall current, which appears at a sample edge and is opposite in direction between two electron layers, we first deplete one electron layer by applying a gate voltage and then observe the DNP in the other layer (occupied by electrons) through the local electrical detection [73-75]. The temporal decay of the DNP is negligible during such a procedure with the gate-voltage application and the electrical detection since the nuclear spin has a long relaxation time of the order of $1000 \mathrm{~s}$.

Now we discuss a measurement procedure to confirm the competition between the pseudospin precession $\omega$ and the momentum relaxation $\tau_{p}^{-1}$. Since it is difficult to change $\omega$ and $\tau_{p}^{-1}$ in a single sample (in experiments [28,31], the tunnel coupling $\hbar \omega$ was changed by varying the $\mathrm{Al}$ concentration and the width of the barrier between two layers), we propose to measure the antiparallel spin Hall conductivity $\sigma_{y x}^{\mathrm{s} 3}$ as a function of the potential offset $\hbar \omega_{V}$ between two layers by means of the gate voltage. From Eq. (67), we obtain

$$
\left(\frac{\sigma_{0}^{\mathrm{sH}}}{\sigma_{y x}^{\mathrm{s} 3}}-1\right)^{-1}=\frac{\tau_{l}}{\omega^{2} \tau_{p}}\left(\omega_{V}^{2}+\tau_{l}^{-2}\right) .
$$

When we plot the observed value of the left-hand side as a function of $\omega_{V}^{2}=\omega_{1}^{2}-\omega^{2}$, the plot should be, according to this formula, a straight line which has a slope of $\tau_{l} / \omega^{2} \tau_{p}$ and intersects the horizontal axis at $-\tau_{l}^{-2}$. Observing such $\omega_{V}$ dependence shows that the competition between the pseudospin precession and the momentum relaxation is relevant to the present crossover [76].

\section{CONCLUSIONS}

We have theoretically investigated the crossover between the decoupled bilayer with antiparallel extrinsic spin Hall current and the strongly coupled bilayer with no spin Hall current as a function of $\Delta_{\text {SAS }}$, the energy separation between the symmetric and antisymmetric states of the motion perpendicular to the layer, and found that the crossover occurs around $\omega\left(\equiv \Delta_{\mathrm{SAS}} / \hbar\right)=\tau_{p}^{-1}$ via the competition between the pseudospin precession with frequency $\omega$ and the momentum relaxation with rate $\tau_{p}^{-1}$. We have successfully explained the present suppression of the antiparallel extrinsic spin Hall current with the increase of $\Delta_{\text {SAS }}$ by extending the Hanle effect dynamics, which was originally written for spin components, to pseudospin components of the spin Hall current. In the pseudospin Hanle effect occurring with the extrinsic spin Hall effect of this bilayer, the momentum relaxation in addition to the pseudospin dephasing plays a role in damping the precession in the pseudospin space of the spin Hall current. Such damping caused by the momentum relaxation is a feature which is absent in the direct pseudospin analog of the Hanle effect.

Although in this paper we have derived the equation describing the pseudospin Hanle effect in a tailor-made double quantum well with the antiparallel extrinsic spin Hall current, the concept of the pseudospin Hanle effect proposed in this paper may generally be applicable to any electron system with the pseudospin degree of freedom, which includes graphene and transition-metal dichalcogenides with the sublattice [77-79] and the valley degree of freedom [78-80], as well as their bilayers with the layer degree of freedom $[77,79,80]$. Then the Hanle effect of the pseudospin may appear in a wide range of phenomena brought by various pseudospins which include many phenomena other than the bilayer spin Hall effect. Thus, we expect that viewing the dynamics involving pseudospin as the Hanle effect will be useful in exploring and explaining many pseudospin-related phenomena as well as in their applications to pseudospintronics $[77,81,82]$ since the Hanle effect for spin has been playing an important role in spin physics and spintronics (see Ref. [4] for a review and [14,15,83-88] for examples of recent achievements).

As mentioned above, the pseudospin Hanle effect of the system studied in this paper has the feature that the momentum relaxation contributes to the damping of the precession in the pseudospin space of the current considered. We expect that such damping due to the momentum relaxation will frequently appear in the dynamics of spin (pseudospin) combined with momentum since the current is used in each process of spintronics (pseudospintronics), such as generation, manipulation, storage, and transport. More precisely, when the Bloch equation is employed for a variable involving both spin (pseudospin) and momentum, it will have a damping term due to the momentum relaxation, in addition to the spin (pseudospin) precession term, which gives rise to the Hanle effect determined by the product of the momentum relaxation time and the spin- (pseudospin-) precession frequency.

\section{ACKNOWLEDGMENT}

This work was supported by Grant-in-Aid for Scientific Research (C) Grant No. JP17K05484 from the Japan Society for the Promotion of Science (JSPS). 
[1] J. Sinova, S. Murakami, S. Q. Shen, and M. S. Choi, Solid State Commun. 138, 214 (2006).

[2] M. I. Dyakonov and A. V. Khaetskii, in Spin Physics in Semiconductors, edited by M. I. Dyakonov (Springer, Berlin, 2008), pp. 211-243.

[3] G. Vignale, J. Supercond. Novel Magn. 23, 3 (2010).

[4] I. Žutić, J. Fabian, and S. D. Sarma, Rev. Mod. Phys. 76, 323 (2004).

[5] S. Murakami, N. Nagaosa, and S.-C. Zhang, Science 301, 1348 (2003).

[6] J. Sinova, D. Culcer, Q. Niu, N. A. Sinitsyn, T. Jungwirth, and A. H. MacDonald, Phys. Rev. Lett. 92, 126603 (2004).

[7] M. I. Dyakonov and V. I. Perel, Sov. Phys. JETP 13, 467 (1971).

[8] M. I. Dyakonov and V. I. Perel, Phys. Lett. A 35, 459 (1971).

[9] J. E. Hirsch, Phys. Rev. Lett. 83, 1834 (1999).

[10] S. Zhang, Phys. Rev. Lett. 85, 393 (2000).

[11] H.-A. Engel, B. I. Halperin, and E. I. Rashba, Phys. Rev. Lett. 95, 166605 (2005).

[12] E. M. Hankiewicz and G. Vignale, Phys. Rev. B 73, 115339 (2006).

[13] W. K. Tse and S. Das Sarma, Phys. Rev. Lett. 96, 056601 (2006).

[14] Y. K. Kato, R. C. Myers, A. C. Gossard, and D. D. Awschalom, Science 306, 1910 (2004).

[15] V. Sih, R. C. Myers, Y. K. Kato, W. H. Lau, A. C. Gossard, and D. D. Awschalom, Nat. Phys. 1, 31 (2005).

[16] S. O. Valenzuela and M. Tinkham, Nature (London) 442, 176 (2006).

[17] N. P. Stern, S. Ghosh, G. Xiang, M. Zhu, N. Samarth, and D. D. Awschalom, Phys. Rev. Lett. 97, 126603 (2006).

[18] T. Kimura, Y. Otani, T. Sato, S. Takahashi, and S. Maekawa, Phys. Rev. Lett. 98, 156601 (2007).

[19] J. Wunderlich, B. Kaestner, J. Sinova, and T. Jungwirth, Phys. Rev. Lett. 94, 047204 (2005).

[20] T. Chakraborty and P. Pietiläinen, Phys. Rev. Lett. 59, 2784 (1987).

[21] D. Yoshioka, A. H. MacDonald, and S. M. Girvin, Phys. Rev. B 39, 1932 (1989).

[22] H. A. Fertig, Phys. Rev. B 40, 1087 (1989).

[23] S. He, X. C. Xie, S. Das Sarma, and F. C. Zhang, Phys. Rev. B 43, 9339 (1991).

[24] Y. W. Suen, L. W. Engel, M. B. Santos, M. Shayegan, and D. C. Tsui, Phys. Rev. Lett. 68, 1379 (1992).

[25] J. P. Eisenstein, G. S. Boebinger, L. N. Pfeiffer, K. W. West, and S. He, Phys. Rev. Lett. 68, 1383 (1992).

[26] X.-G. Wen and A. Zee, Phys. Rev. Lett. 69, 1811 (1992).

[27] Z. F. Ezawa and A. Iwazaki, Phys. Rev. B 47, 7295 (1993).

[28] G. S. Boebinger, H. W. Jiang, L. N. Pfeiffer, and K. W. West, Phys. Rev. Lett. 64, 1793 (1990).

[29] Y. W. Suen, J. Jo, M. B. Santos, L. W. Engel, S. W. Hwang, and M. Shayegan, Phys. Rev. B 44, 5947 (1991).

[30] G. S. Boebinger, L. N. Pfeiffer, and K. W. West, Phys. Rev. B 45, 11391 (1992).

[31] S. Q. Murphy, J. P. Eisenstein, G. S. Boebinger, L. N. Pfeiffer, and K. W. West, Phys. Rev. Lett. 72, 728 (1994).

[32] A. H. MacDonald, P. M. Platzman, and G. S. Boebinger, Phys. Rev. Lett. 65, 775 (1990).

[33] L. Brey, Phys. Rev. Lett. 65, 903 (1990).

[34] Since the electron correlation is not important in the absence of the Landau quantization, the change between the presence and absence of the extrinsic spin Hall effect occurs in the form of a crossover.

[35] R. J. Haug, R. R. Gerhardts, K. von Klitzing, and K. Ploog, Phys. Rev. Lett. 59, 1349 (1987).

[36] N. F. Mott, Proc. R. Soc. A 124, 425 (1929).

[37] J. Smit, Physica 21, 877 (1955).

[38] J. Smit, Physica 24, 39 (1958).

[39] H. Akera and H. Suzuura, Phys. Rev. B 87, 075301 (2013).

[40] M. I. Dyakonov and V. I. Perel, Sov. Phys. JETP 33, 1053 (1971).

[41] M. I. Dyakonov and V. I. Perel, Sov. Phys. Solid State 13, 3023 (1972).

[42] M. I. Dyakonov and V. Y. Kachorovskii, Sov. Phys. Semicond. 20, 110 (1986).

[43] W. Hanle, Z. Phys. 30, 93 (1924).

[44] R. R. Parsons, Phys. Rev. Lett. 23, 1152 (1969).

[45] M. I. Dyakonov and V. I. Perel, in Optical Orientation, edited by F. Meier and B. P. Zakharchenya (North Holland, Amsterdam, 1984), pp. 11-71.

[46] P. Nozières and C. Lewiner, J. Phys. (Paris) 34, 901 (1973).

[47] R. Lassnig, Phys. Rev. B 31, 8076 (1985).

[48] R. S. Calsaverini, E. Bernardes, J. C. Egues, and D. Loss, Phys. Rev. B 78, 155313 (2008).

[49] F. J. Ohkawa and Y. Uemura, J. Phys. Soc. Jpn. 37, 1325 (1974).

[50] Y. A. Bychkov and E. I. Rashba, J. Phys. C 17, 6039 (1984).

[51] Y. A. Bychkov and E. I. Rashba, JETP Lett. 39, 78 (1984).

[52] G. Dresselhaus, Phys. Rev. 100, 580 (1955).

[53] W. Kohn and J. M. Luttinger, Phys. Rev. 108, 590 (1957).

[54] L. Berger, Phys. Rev. B 2, 4559 (1970).

[55] L. Berger, Phys. Rev. B 5, 1862 (1972).

[56] S. K. Lyo and T. Holstein, Phys. Rev. Lett. 29, 423 (1972).

[57] D. Culcer, E. M. Hankiewicz, G. Vignale, and R. Winkler, Phys. Rev. B 81, 125332 (2010).

[58] N. Nagaosa, J. Sinova, S. Onoda, A. H. MacDonald, and N. P. Ong, Rev. Mod. Phys. 82, 1539 (2010).

[59] M. I. Dyakonov and A. V. Khaetskii, Sov. Phys. JETP 59, 1072 (1984).

[60] X. Hu, R. De Sousa, and S. Das Sarma, in Foundations of Quantum Mechanics in the Light of New Technology, edited by Y. A. Ono and K. Fujikawa (World Scientific, Singapore, 2002), pp. 3-11.

[61] E. L. Ivchenko, Sov. Phys. Solid State 15, 1048 (1973).

[62] M. M. Glazov and E. Ivchenko, J. Supercond. 16, 735 (2003).

[63] E. G. Mishchenko and B. I. Halperin, Phys. Rev. B 68, 045317 (2003).

[64] E. M. Hankiewicz, L. W. Molenkamp, T. Jungwirth, and J. Sinova, Phys. Rev. B 70, 241301(R) (2004).

[65] C. Brüne, A. Roth, E. G. Novik, M. König, H. Buhmann, E. M. Hankiewicz, W. Hanke, J. Sinova, and L. W. Molenkamp, Nat. Phys. 6, 448 (2010).

[66] P. Wadley, B. Howells, J. Železný, C. Andrews, V. Hills, R. P. Campion, V. Novák, K. Olejník, F. Maccherozzi, S. S. Dhesi, S. Y. Martin, T. Wagner, J. Wunderlich, F. Freimuth, Y. Mokrousov, J. Kuneš, J. S. Chauhan, M. J. Grzybowski, A. W. Rushforth, K. W. Edmonds, B. L. Gallagher, and T. Jungwirth, Science 351, 587 (2016).

[67] T. Jungwirth, X. Marti, P. Wadley, and J. Wunderlich, Nat. Nanotechnol. 11, 231 (2016).

[68] S. Hidaka, M. Akabori, and S. Yamada, Appl. Phys. Express 5, 113001 (2012). 
[69] F. Dettwiler, J. Fu, S. Mack, P. J. Weigele, J. C. Egues, D. D. Awschalom, and D. M. Zumbühl, Phys. Rev. X 7, 031010 (2017).

[70] H. Akera, H. Suzuura, and Y. Egami, Phys. Rev. B 92, 205311 (2015).

[71] C. J. Trowbridge, B. M. Norman, Y. K. Kato, D. D. Awschalom, and V. Sih, Phys. Rev. B 90, 085122 (2014).

[72] J. Strand, A. F. Isakovic, X. Lou, P. A. Crowell, B. D. Schultz, and C. J. Palmstrøm, Appl. Phys. Lett. 83, 3335 (2003).

[73] K. R. Wald, L. P. Kouwenhoven, P. L. McEuen, N. C. van der Vaart, and C. T. Foxon, Phys. Rev. Lett. 73, 1011 (1994).

[74] T. Machida, T. Yamazaki, and S. Komiyama, Appl. Phys. Lett. 80, 4178 (2002).

[75] Y. Komori, S. Sakuma, and T. Okamoto, Phys. Rev. Lett. 99, 146807 (2007).

[76] Experimentally, the value of $\Delta_{\text {SAS }}$ can be changed in the range of $0<\Delta_{\text {SAS }}<1 \mathrm{meV}[28,31]$. The value of the momentum relaxation time is $\tau_{p}=0.1 \mathrm{ps}$ in the sample used for the observation of the spin Hall effect in a quantum well [15] and can be increased by, at least, three orders of magnitude in high-mobility samples. $\Delta_{\text {SAS }} \tau_{p} / \hbar=\omega \tau_{p} \sim 1$ when $\Delta_{\text {SAS }}=$ $1 \mathrm{meV}$ and $\tau_{p}=1 \mathrm{ps}$. The potential offset with a magnitude of $\hbar \omega_{V}=1 \mathrm{meV}$ between two wells with a distance of $10 \mathrm{~nm}$ is produced by the applied electric field of $10^{5} \mathrm{~V} / \mathrm{m}$. Such values of the applied electric field are experimentally accessible in quantum well structures by using a gate electrode (see, for example, Ref. [89]).
[77] D. Pesin and A. H. MacDonald, Nat. Mater. 11, 409 (2012).

[78] T. Ando, J. Phys. Soc. Jpn. 84, 114705 (2015).

[79] T. Ando, J. Phys. Soc. Jpn. 84, 114704 (2015).

[80] X. Xu, W. Yao, D. Xiao, and T. F. Heinz, Nat. Phys. 10, 343 (2014).

[81] H. Min, G. Borghi, M. Polini, and A. H. MacDonald, Phys. Rev. B 77, 041407(R) (2008).

[82] P. San-Jose, E. Prada, E. McCann, and H. Schomerus, Phys. Rev. Lett. 102, 247204 (2009).

[83] N. S. Averkiev, L. E. Golub, A. S. Gurevich, V. P. Evtikhiev, V. P. Kochereshko, A. V. Platonov, A. S. Shkolnik, and Y. P. Efimov, Phys. Rev. B 74, 033305 (2006).

[84] C. Józsa, T. Maassen, M. Popinciuc, P. J. Zomer, A. Veligura, H. T. Jonkman, and B. J. van Wees, Phys. Rev. B 80, 241403(R) (2009).

[85] S. P. Dash, S. Sharma, J. C. Le Breton, J. Peiro, H. Jaffrès, J.-M. George, A. Lemaître, and R. Jansen, Phys. Rev. B 84, 054410 (2011).

[86] S. Omar and B. J. van Wees, Phys. Rev. B 95, 081404(R) (2017).

[87] A. Dankert and S. P. Dash, Nat. Commun. 8, 16093 (2017).

[88] Y. K. Luo, J. Xu, T. Zhu, G. Wu, E. J. McCormick, W. Zhan, M. R. Neupane, and R. K. Kawakami, Nano Lett. 17, 3877 (2017).

[89] G. Engels, J. Lange, T. Schäpers, and H. Lüth, Phys. Rev. B 55, R1958 (1997). 